\title{
Al et réinsertion: il faut de véritables incitatifs pour les employeurs
}

«Attention! Les institutions de la sécurité sociale peuvent compromettre votre santé!» déclara Eva Nadai, professeure de sociologie à la Haute école de Suisse du nord-ouest, en introduction à son exposé présenté le 9 septembre 2010 au congrès de Public Health Suisse. Et d'ajouter que depuis près de 20 ans, les institutions de la sécurité sociale cherchent de moins en moins à garantir un revenu de remplacement mais bien plutôt à exploiter à tout prix la «capacité résiduelle de travail», ce qui, selon elle, occasionne chez les personnes concernées un stress important et nuisible pour la santé.

On tente aussi de nous persuader que la $6^{\mathrm{e}}$ révision de l'AI est une révision orientée sur la réinsertion professionnelle: «La révision des rentes axée sur la réadaptation réduit le nombre de rentes d'environ 5\%. Pour atteindre ce but, la réadaptation des personnes qui ont de réelles chances d'être réinsérées sera activement encouragée.» [1].

\section{Pas de réinsertion professionnelle à tout prix}

La lettre d'accompagnement à la procédure de consultation pour le deuxième paquet de mesures de la $6^{\mathrm{e}}$ révision $\mathrm{AI}$ le répète: «Renforcement de la réadaptation et maintien sur le marché du travail: les instruments mis en place par la $5^{\mathrm{e}}$ révision seront optimisés et développés.» [2].

Mais à quoi sert la réinsertion à tout prix? Selon Eva Nadai, des études internationales montrent que ces tentatives ne fonctionnent pas. En effet, elles n'ont guère d'effet sur l'emploi et n'apportent que peu de résultats dans la lutte contre la pauvreté.

\section{Les employeurs doivent être associés de manière efficace à la réinsertion professionnelle}

Revenons quelques décennies en arrière: lorsque l'AI fut introduite, on voyait en elle «surtout (...) un moyen de mobiliser des travailleurs peu qualifiés, dont l'économie suisse, florissante à l'époque, avait grandement besoin...» [3]. Mais la situation est aujourd'hui totalement différente: les entreprises peuvent chercher sans autres dans l'Union européenne des employés qualifiés, en bonne santé et donc productifs; en outre, elles ont tendance, sous la forte pression exercée par la concurrence et la recherche de l'efficacité, à licencier ou à simplement ne pas engager de personnes partiellement handicapées et peut-être aussi mal qualifiées.
De ce fait, la réinsertion nécessite des incitatifs vraiment efficaces et significatifs pour les employeurs [4]. Ce qui a été proposé jusqu'à présent ne suffit pas.

Et enfin, la proposition de faire évaluer la capacité fonctionnelle à travailler de la personne assurée exclusivement par les services médicaux régionaux (SMR) est inadmissible. Les responsables de la réinsertion professionnelle ne se réfèrent plus au seul jugement de Dieu, comme au Moyen Age, mais recourent à toutes les ressources disponibles, dont fait également partie le médecin traitant qui, souvent, accompagne le patient depuis des années!

\section{Il ne faut pas que les services médicaux régionaux soient les seuls à évaluer la capacité à travailler}

La FMH est volontiers prête à soutenir les efforts faits pour toute réinsertion judicieuse. Mais la collaboration des médecins traitants et une implication vraie et efficace des employeurs sont indispensables. Or la révision actuelle ne prévoit ni l'un ni l'autre, comme l'a souligné la FMH dans sa prise de position.

«More of the same» n'est un produit miracle ni en médecine ni en politique. En Suisse, nous nous attelons à la $6^{\mathrm{e}}$ révision de l'AI sans savoir si les mesures onéreuses de réinsertion prévues par la $5^{\mathrm{e}}$ révision de l'AI sont vraiment efficaces.

Dr Christine Romann, Membre du Comité central de la FMH, Responsable du domaine Promotion de la santé et prévention

Hanspeter Kuhn, avocat, Secrétaire général adjoint de la FMH

1 Assurance-invalidité - $6^{\mathrm{e}}$ révision de l'AI, premier paquet de mesures, rapport explicatif, 17 juin 2009.

2 Assurance-invalidité - $6^{\text {e }}$ révision de l'AI, deuxième paquet de mesures (révision 6b), 25 juin 2010.

3 Germann U. AI: une mise en œuvre après une longue maturation. Sécurité sociale CHSS 1/2010, p. 5 à 8 : «Les travaux préparatoires de l'AI furent marqués par le ‘boom de la réadaptation`, apparu à la fin des années 40. Le «don suisse pour la fête nationale» de 1947 avait déjà été consacré à la réadaptation des infirmes. (...) Ainsi, la FSIH voyait surtout dans la réadaptation des handicapés un moyen de mobiliser des travailleurs peu qualifiés, dont l'économie suisse, florissante à l'époque, avait grandement besoin et qu'elle devait généralement recruter dans les pays européens.»

4 Des réglementations de quota, comme les organisations d'handicapés le réclament, seraient certainement aussi possibles; elles promeuvent toutefois l'Etat-surveillant. 